\title{
Analysis of risk factors for a high prevalence of extended-spectrum $\beta$-lactamase-producing Enterobacteriaceae in asymptomatic individuals in rural Thailand
}

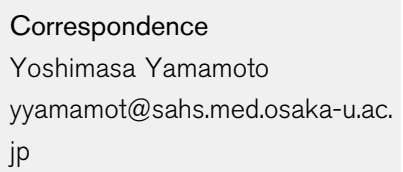

Received 7 October 2010 Accepted 28 January 2011

\author{
Ulzii-Orshikh Luvsansharav, ${ }^{1}$ Itaru Hirai, ${ }^{1,2}$ Marie Niki, ${ }^{1}$ Tadahiro Sasaki, ${ }^{1}$ \\ Kiyoko Makimoto, ${ }^{3}$ Chalit Komalamisra, ${ }^{4}$ Wanna Maipanich, ${ }^{4}$ \\ Teera Kusolsuk, ${ }^{4}$ Surapol Sa-Nguankiat, ${ }^{4}$ Somchit Pubampen ${ }^{4}$ \\ and Yoshimasa Yamamoto ${ }^{1,2}$ \\ ${ }^{1}$ Department of Bioinformatics, Osaka University Graduate School of Medicine, Osaka, Japan \\ ${ }^{2}$ International Center for Medical Research and Treatment, Kobe University Graduate School of \\ Medicine, Kobe, Japan \\ ${ }^{3}$ Department of Nursing, Osaka University Graduate School of Medicine, Osaka, Japan \\ ${ }^{4}$ Department of Helminthology, Faculty of Tropical Medicine, Mahidol University, Bangkok, Thailand
}

The prevalence of and risk factors associated with extended-spectrum $\beta$-lactamase (ESBL)producing micro-organisms have not been well studied in healthy individuals. The aim of this study was to determine this in healthy individuals in Thailand. Stool samples and questionnaires obtained from 445 participants from three provinces in Thailand were analysed. The antimicrobial susceptibility of the isolates was assessed using phenotypic and genotypic methods. PCR analysis was performed to detect and group the bla СTX-M genes. The prevalence of CTX-M-type ESBL-producing Enterobacteriaceae in the three provinces was as follows: $29.3 \%$ in Nan (43/147), 29.9\% in Nakhon Si Thammarat (43/144) and 50.6\% in Kanchanaburi (78/154) $(P<0.001)$. Of the 445 samples, $33(7.4 \%), 1(0.2 \%)$ and $127(28.5 \%)$ isolates belonged to the

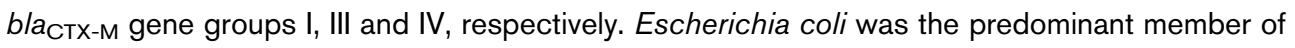
the Enterobacteriaceae producing CTX-M-type ESBLs (40/43, 39/43 and 70/78 isolates in Nan, Nakhon Si Thammarat and Kanchanaburi, respectively). No statistically significant association was observed between the presence of ESBL-producing bacteria and gender, age, education, food habits or antibiotic usage. However, the provinces that had the highest prevalence of ESBLproducing Enterobacteriaceae also had the highest prevalence of use and purchase of antibiotics without a prescription. Thus, this study revealed that faecal carriage of ESBL-producing Enterobacteriaceae is very high in asymptomatic individuals in Thailand, with some variations among the provinces. This high prevalence may be linked to antibiotic abuse.

\section{INTRODUCTION}

Multidrug-resistant Enterobacteriaceae that produce extendedspectrum $\beta$-lactamases (ESBLs) have spread rapidly worldwide causing treatment failure and hindering infection control efforts (Paterson \& Bonomo, 2005; Pitout \& Laupland, 2008). ESBLs are $\beta$-lactamases capable of inducing resistance to penicillins and to first-, second- and third-generation cephalosporins and monobactams but not to cephamycins and carbapenems (Paterson \& Bonomo, 2005; Pitout \& Laupland, 2008; Rodríguez-Baño \& Pascual, 2008). Furthermore, the wide spread of ESBL-producing bacteria

Abbreviation: ESBL, extended-spectrum $\beta$-lactamase. not only affects the choice of antimicrobials but may also cause excessive morbidity and mortality, as well as increased healthcare costs (Roberts et al., 2009).

Among the ESBLs, the CTX-M-type enzymes are more prevalent, and Escherichia coli strains that produce CTX-Mtype $\beta$-lactamases are frequently true community-acquired ESBL-producing pathogens (Pitout \& Laupland, 2008). These E. coli strains cause community-acquired urinary tract and bloodstream infections (Ben-Ami et al., 2009; RodríguezBaño et al., 2006). The prevalence of and risk factors associated with the carriage of ESBL-producing microorganisms have often been studied in patients with these infections. This prevalence varies among countries, and risk 
factor analyses have shown conflicting and varying results (Ben-Ami et al., 2009; Coque et al., 2008; Lautenbach et al., 2001; Paterson \& Bonomo, 2005). In contrast, only a few studies have been performed on the community carriage of ESBL-producing E. coli. In recent years, researchers have reported a 2.3, 3.7, 7.0 and $13.1 \%$ prevalence of ESBLproducing Enterobacteriaceae in healthy subjects in Lebanon, Spain, China and Saudi Arabia, respectively (Kader et al., 2007; Moubareck et al., 2005; Tian et al., 2008; Valverde et al., 2004). However, in a previous study by us, we observed a surprisingly high prevalence of CTX-M-type ESBL-producing Enterobacteriaceae (82/160 samples) in asymptomatic individuals in Kanchanaburi province, Thailand (Sasaki et al., 2010). Therefore, the aim of the present study was to analyse the factors that may have contributed to the extensive spread of ESBL-producing Enterobacteriaceae in asymptomatic individuals in Thailand. We extended our study beyond the hospital environment and performed an epidemiological analysis of the risk factors in order to predict the possible threat of the spread of ESBL-producing Enterobacteriaceae.

\section{METHODS}

Location of provinces. The study was conducted from May to July 2009 in Nan and Nakhon Si Thammarat provinces in Thailand. Epidemiological data from a previous study in Kanchanaburi province, Thailand, were also used in this study (Sasaki et al., 2010). The three provinces were selected to represent geographically different regions of the country: Nan is in the northern region, Nakhon Si Thammarat is in the southern region and Kanchanaburi is in the central region (Fig. 1). Written informed consent was obtained from each individual participating in the study.

Study samples. We analysed a total of 468 stool samples from the three Thai provinces: one sample was obtained from each asymptomatic volunteer. The participants were screened on the basis of age $(\geqslant 20$ years $)$ and medical history. Asymptomatic individuals with a history of antibiotic treatment 3 months prior to sample collection and with a confirmed diagnosis of digestive tract diseases were excluded from the study.

Questionnaire. All participants were interviewed using a standardized questionnaire based on general demographic and socioeconomic circumstances. They were questioned about their food intake, drinking water resources and toilet facilities. The participants were also questioned regarding their alcohol intake and smoking habits. We questioned them about their antibiotic usage, including the purchase of antibiotics without a prescription. After excluding the questionnaires in which age and gender data had not been filled in, we used 445 questionnaires (183 men and 262 women) for statistical analyses. In Kanchanaburi, four of the excluded participants had ESBL-producing Enterobacteriaceae.

Screening tests for ESBLs. Stool samples were collected from asymptomatic participants to identify ESBL-producing Enterobacteriaceae by phenotypic and genotypic methods. The phenotypic methods included plating the stool samples on MacConkey agar supplemented with $2 \mu \mathrm{g}$ cefotaxime $\mathrm{ml}^{-1}$ within $24 \mathrm{~h}$ after collection. Subsequently, the results were confirmed by a double-disc synergy test using amoxicillin-clavulanate and cefotaxime, ceftazidime, cefepime and aztreonam, as described previously (Drieux et al., 2008; Sasaki et al., 2010). Isolates were identified using conventional biochemical tests.

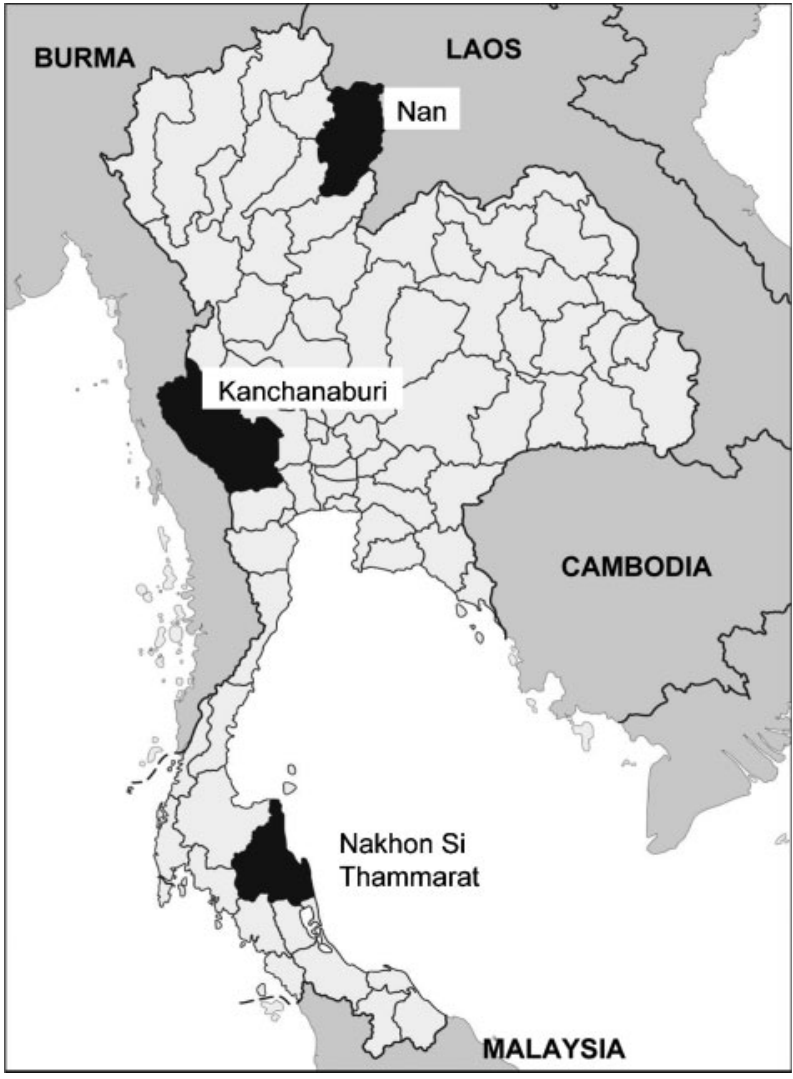

Fig. 1. Location of the Thai provinces selected for the study.

DNA extraction and PCR analysis. Bacterial DNA was extracted from the isolates by boiling the bacterial suspensions. DNA samples with a concentration of $0.1 \mathrm{ng} \mu \mathrm{l}^{-1}$ were used as template for PCR analysis. The universal primers CTX-M-U1 (5'-ATGTGCAGYACCAGTAARGTKATGGC-3') and CTX-M-U2 (5'-TGGGTRAARTARGTSACCAGAAYCAGCGG- $\left.3^{\prime}\right)$ were used to detect the $b l a_{\text {CTX-M }}$ gene, as described previously (Monstein et al., 2007). DNA from the reference

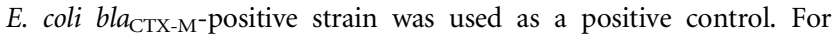
genotyping the $b l a_{\text {СTX-M }}$ genes, we used four primer sets that amplify group-specific bla $a_{\mathrm{CTX}-\mathrm{M}}$ genes, as described elsewhere (Pitout et al., 2004): group I, CTX-M-1, CTX-M-3, CTX-M-10-CTX-M-12, CTX-M15, CTX-M-22, CTX-M-23, CTX-M-28, CTX-M-29 and CTX-M-30; group II, CTX-M-2, CTX-M-4-CTX-M-7, CTX-M-20 and Toho-1; group III, CTX-M-8; and group IV, CTX-M-9, CTX-M-13, CTX-M-14, CTX-M-16-CTX-M-19, CTX-M-21, CTX-M-27 and Toho-2. The PCR products were visualized by $2 \%$ agarose gel electrophoresis followed by staining with GelRed Nucleic Acid Gel Stain (Biotium).

Statistical analysis. Statistical analysis was performed using SPSS v.16.0. Data from the three provinces were examined separately for each province and for all provinces together. All variables were analysed by univariate analysis using a $\chi^{2}$ test or Fisher's exact test, as appropriate. Statistical significance was set at $P<0.05$.

\section{RESULTS}

The characteristics of the 445 study participants from Nan, Nakhon Si Thammarat and Kanchanaburi provinces in Thailand are listed in Table 1. The median age of the study 
Table 1. Characteristics of the study participants

Missing data were excluded from the analysis.

\begin{tabular}{|lccc|}
\hline Variable & \multicolumn{3}{c|}{ Province } \\
\cline { 2 - 4 } & Nan $(\boldsymbol{n}=\mathbf{1 4 7})$ & $\begin{array}{c}\text { Nakhon Si Thammarat } \\
(\boldsymbol{n}=\mathbf{1 4 4})\end{array}$ & $\begin{array}{c}\text { Kanchanaburi } \\
(\boldsymbol{n}=\mathbf{1 5 4})\end{array}$ \\
\hline Age (years): median (range) & $48(30-71)$ & $59(39-86)$ & $55(25-86)$ \\
Gender: male sex & $70(48 \%)$ & $53(37 \%)$ & $60(39 \%)$ \\
Education: no formal education & $55(38 \%)$ & $10(7 \%)$ & $48(32 \%)$ \\
Occupation: farmer & $132(92 \%)$ & $89(64 \%)$ & $68(61 \%)$ \\
Lifestyle & $72(50 \%)$ & $23(18 \%)$ & $66(45 \%)$ \\
$\quad$ Current alcohol use & $19(13 \%)$ & $33(23 \%)$ & $145(94 \%)$ \\
Current smoking $\dagger$ & $138(94 \%)$ & $127(88 \%)$ & $106(70 \%)$ \\
$\quad$ Meat consumption $\neq$ & $54(37 \%)$ & $51(36 \%)$ & $31(20 \%)$ \\
Housing condition & $19(13 \%)$ & $47(33 \%)$ & \\
Tap water at home & & & \\
Flush toilet at home & & & \\
\end{tabular}

*Subjects who drink alcoholic beverages one or more times a month.

†Current smoking includes both daily and non-daily or occasional smoking.

$\ddagger$ Subjects who consume meat one or more times a week.

participants was 54 years (range 25-86 years). The study participants were from the rural areas of Thailand and were predominantly farmers $(72 \%)$. Sixty-two per cent of the participants had received primary school education, whilst $26 \%$ had not received any formal education.

The double-disc synergy test revealed that $32.0 \%(47 / 147$ samples), $32.6 \%$ (47/144 samples) and $53.9 \%(83 / 154$ samples) of the isolates in the Nan, Nakhon Si Thammarat and Kanchanaburi provinces, respectively, were ESBLproducing bacteria. The prevalence of CTX-M-type ESBLproducing Enterobacteriaceae, as confirmed by phenotypic and genotypic methods, was $29.3 \%$ in the Nan province
(43/147 samples), $29.9 \%$ in the Nakhon Si Thammarat province (43/144 samples) and $50.6 \%$ in the Kanchanaburi province (78/154 samples) (Fig. 2). The prevalence in Kanchanaburi was significantly higher than that in the other two provinces $(P<0.001)$. Genotyping of $b l a_{\text {CTX-M }}$ gene-positive isolates revealed that $33(7.4 \%), 1(0.2 \%)$ and $127(28.5 \%)$ of the 445 samples belonged to groups I, III and IV, respectively (Fig. 2). Three of the isolates did not belong to any of the groups tested. On performing bacterial identification for CTX-M-type ESBL-producing Enterobacteriaceae, we found that E. coli was the predominant CTX-M-type ESBL-producing member of the Enterobacteriaceae in these isolates (40/43 isolates in Nan,

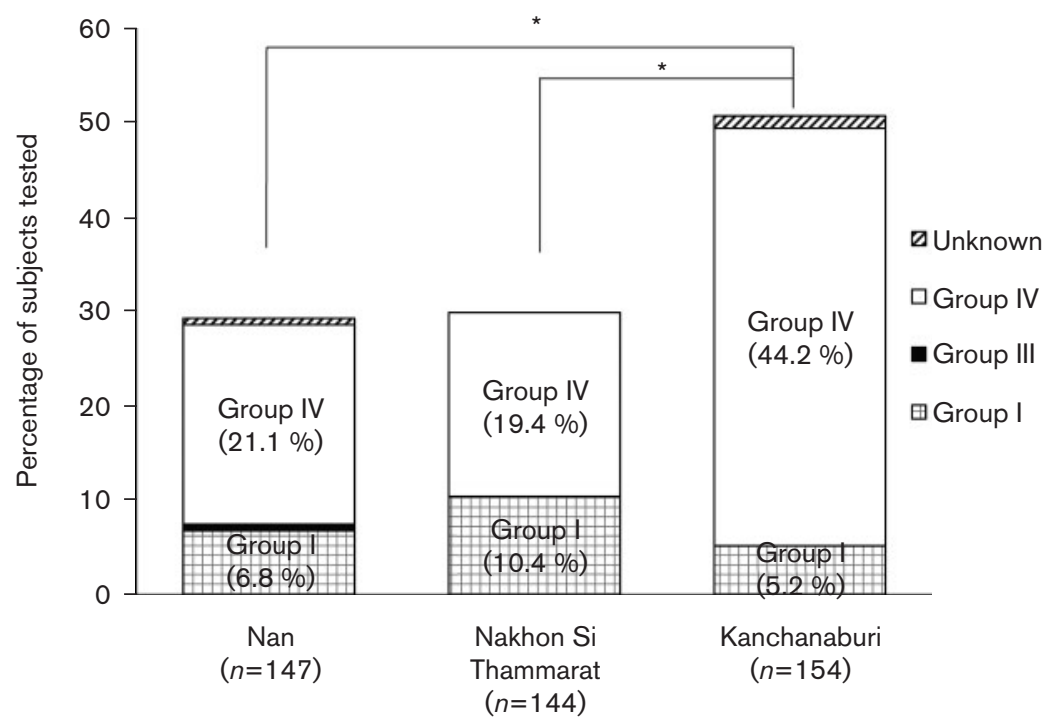

Fig. 2. Prevalence of CTX-M-type ESBLproducing Enterobacteriaceae and b/a ${ }_{\mathrm{CTX}-\mathrm{M}}$ gene groups. ${ }^{\star} P<0.001$; prevalence was compared between the provinces using a $\chi^{2}$ test. 


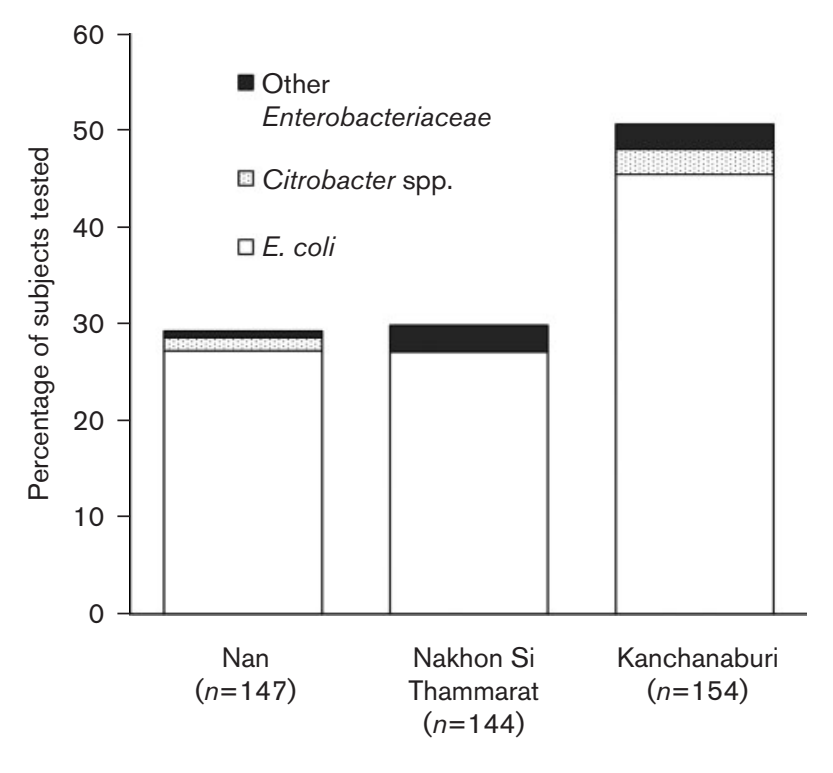

Fig. 3. Bacterial identification of CTX-M-type ESBL-producing Enterobacteriaceae.

39/43 isolates in Nakhon Si Thammarat and 70/78 isolates in Kanchanaburi) (Fig. 3). The other CTX-M-type ESBLproducing bacterial species isolated were Citrobacter, Klebsiella and Enterobacter.

No statistically significant association was observed between the presence of ESBL-producing bacteria and gender, age, education and food habits of or antibiotic usage by the participants (data not shown). However, on comparing antibiotic usage in the three provinces, we observed a significant difference between the three provinces. In particular, the pattern of antibiotic purchase without a prescription was similar to the pattern of the prevalence of ESBL-producing Enterobacteriaceae in these provinces (Table 2 ).

\section{DISCUSSION}

In this study, the prevalence of CTX-M-type ESBLproducing Enterobacteriaceae in asymptomatic Thai individuals (29.3-50.6\%) was alarmingly high compared with that observed in previous studies performed on healthy subjects; these studies reported a $2.4-13.1 \%$ prevalence of faecal carriage of ESBL-producing Enterobacteriaceae in different countries (Kader et al., 2007; Moubareck et al., 2005; Tian et al., 2008; Valverde et al., 2004). A recent publication by Lo et al. (2010) reported $50.3 \%$ faecal carriage of ESBL-producing bacteria in household adults of children admitted to a paediatric department with mild febrile illness. In contrast, our study was conducted in community settings among healthy subjects.

The faecal carriage of ESBL-producing Enterobacteriaceae in asymptomatic Thai individuals is close to or sometimes higher than the prevalence of ESBL-producing bacteria in clinical isolates collected from patients in Thailand (30-48 \% in 2005-2007; Hawser et al., 2009; Kiratisin et al., 2008b). Lactamases belonging to CTX-M group I (20\%) and IV (77\%) were predominant among the 164 CTX-M $\beta$-lactamases identified in asymptomatic individuals; this pattern is identical to that of patient isolates in Thailand, where CTX-M-14 (group IV) and CTX-M-15 (group I) were the ESBLs mainly responsible for resistance in E. coli (Kiratisin et al., 2008a; Niumsup et al., 2008). Our findings suggest that ESBL-producing bacterial isolates obtained from clinical specimens in hospital settings represent only a fraction of the actual prevalence, and that a much more rapid and broader community-wide spread of ESBLproducing bacteria may already be under way. Therefore, an increase in the prevalence of ESBL-producing microorganisms among patients is to be expected in the future.

Even though the presence of ESBL-producing microorganisms was not associated with any of the risk factors examined in this study, the comparison of antibiotic usage between the provinces studied suggested that unregulated

Table 2. Comparison of antibiotic usage in the three provinces during the last year

\begin{tabular}{|lcccc|}
\hline Variable & \multicolumn{3}{c}{ Province } & \multirow{2}{*}{ v value $^{*}$} \\
\cline { 2 - 4 } & Nan $(\boldsymbol{n}=\mathbf{1 4 7})$ & $\begin{array}{c}\text { Nakhon Si } \\
\text { Thammarat }(\boldsymbol{n}=\mathbf{1 4 4})\end{array}$ & $\begin{array}{c}\text { Kanchanaburi } \\
(\boldsymbol{n}=\mathbf{1 5 4}) \dagger\end{array}$ & \\
\hline Antibiotic usage & & & & $<0.001$ \\
Yes & $65(44.2 \%)$ & $94(65.3 \%)$ & $104(67.5 \%)$ & \\
No & $81(55.1 \%)$ & $48(33.3 \%)$ & $44(28.6 \%)$ & \\
Unknown or missing & $1(0.7 \%)$ & $2(1.4 \%)$ & $6(3.9 \%)$ & 0.006 \\
Used and purchased antibiotics & & & & \\
Without a prescription & $34(23.1 \%)$ & $34(23.6 \%)$ & $62(40.3 \%)$ & \\
With a prescription & $30(20.4 \%)$ & $56(38.9 \%)$ & $40(26.0 \%)$ & \\
Unknown or missing & $1(0.7 \%)$ & $4(2.8 \%)$ & $2(1.3 \%)$ & \\
\hline
\end{tabular}

${ }^{\star}$ Calculated by $\chi^{2}$ test. Missing or unknown data were excluded from the $\chi^{2}$ test.

$\dagger$ Data for Kanchanaburi province were obtained from a previous study conducted by Sasaki et al. (2010). 
and high consumption of antibiotics may be a risk factor for the high prevalence of ESBL-producing Enterobacteriaceae. In this regard, previous studies have reported correlations between antimicrobial resistance and antibiotic usage (Goossens et al., 2005; Tian et al., 2008). The reason that our analysis did not show a direct association between the presence of ESBL-producing bacteria and antibiotic usage could be that individuals who had recently undergone antibiotic therapy had been excluded.

Furthermore, several studies have linked the high prevalence of antibiotic-resistant bacteria in the human population to the use of antibiotics in the farming industry (Bailar \& Travers, 2002; Dutil et al., 2010; Hawkey \& Jones, 2009; Larson, 2007). Recent studies have revealed an increased prevalence of ESBL-producing micro-organisms in poultry at chicken farms (Briñas et al., 2005; Yuan et al., 2009). Other studies have also reported that farmers may be more likely than the general population to acquire antibiotic-resistant bacteria of food-animal origin (AubryDamon et al., 2004; Swartz, 2002). However, the current study did not reveal any direct association between the prevalence of ESBL-producing bacteria and meat consumption or the occupation of the participants. This may be because of the homogeneity in the population of farmers who participated in the study or because most of them only had backyard chickens and not big industrialized farms. Moreover, studies indicate that contamination of the environment by large amounts of antimicrobials and heavy metal pollution may also select for antibiotic resistance in nature and favour the dissemination of antibiotic resistance genes (Martínez, 2008). Therefore, in order to clarify these issues, environmental data collection should be considered for future studies.

In summary, faecal carriage of ESBL-producing Enterobacteriaceae among asymptomatic individuals in Thailand is very high, with some variations in the prevalence among the provinces. Epidemiological analysis suggested that the high prevalence of ESBL-producing Enterobacteriaceae may be linked to antibiotic abuse.

\section{ACKNOWLEDGEMENTS}

This work was supported by grants from the Ministry of Education, Culture, Sports, Science and Technology, Japan. The excellent technical assistance of Kozue Moriai and Mark Dionisio Francisco is acknowledged.

\section{REFERENCES}

Aubry-Damon, H., Grenet, K., Sall-Ndiaye, P., Che, D., Cordeiro, E., Bougnoux, M. E., Rigaud, E., Le Strat, Y., Lemanissier, V. \& other authors (2004). Antimicrobial resistance in commensal flora of pig farmers. Emerg Infect Dis 10, 873-879.

Bailar, J. C., III \& Travers, K. (2002). Review of assessments of the human health risk associated with the use of antimicrobial agents in agriculture. Clin Infect Dis 34 (Suppl. 3), S135-S143.
Ben-Ami, R., Rodríguez-Baño, J., Arslan, H., Pitout, J. D., Quentin, C., Calbo, E. S., Azap, O. K., Arpin, C., Pascual, A. \& other authors (2009). A multinational survey of risk factors for infection with extended-spectrum $\beta$-lactamase-producing Enterobacteriaceae in nonhospitalized patients. Clin Infect Dis 49, 682-690.

Briñas, L., Moreno, M. A., Teshager, T., Sáenz, Y., Porrero, M. C., Dominguez, L. \& Torres, C. (2005). Monitoring and characterization of extended-spectrum $\beta$-lactamases in Escherichia coli strains from healthy and sick animals in Spain in 2003. Antimicrob Agents Chemother 49, 1262-1264.

Coque, T. M., Baquero, F. \& Canton, R. (2008). Increasing prevalence of ESBL-producing Enterobacteriaceae in Europe. Euro Surveill 13, 19044.

Drieux, L., Brossier, F., Sougakoff, W. \& Jarlier, V. (2008). Phenotypic detection of extended-spectrum $\beta$-lactamase production in Enterobacteriaceae: review and bench guide. Clin Microbiol Infect 14 (Suppl. 1), 90-103.

Dutil, L., Irwin, R., Finley, R., Ng, L. K., Avery, B., Boerlin, P., Bourgault, A. M., Cole, L., Daignault, D. \& other authors (2010). Ceftiofur resistance in Salmonella enterica serovar Heidelberg from chicken meat and humans, Canada. Emerg Infect Dis 16, 48-54.

Goossens, H., Ferech, M., Vander Stichele, R., Elseviers, M. \& ESAC Project Group (2005). Outpatient antibiotic use in Europe and association with resistance: a cross-national database study. Lancet $365,579-587$.

Hawkey, P. M. \& Jones, A. M. (2009). The changing epidemiology of resistance. J Antimicrob Chemother 64 (Suppl. 1), i3-i10.

Hawser, S. P., Bouchillon, S. K., Hoban, D. J., Badal, R. E., Hsueh, P. R. \& Paterson, D. L. (2009). Emergence of high levels of extendedspectrum- $\beta$-lactamase-producing Gram-negative bacilli in the AsiaPacific region: data from the Study for Monitoring Antimicrobial Resistance Trends (SMART) program, 2007. Antimicrob Agents Chemother 53, 3280-3284.

Kader, A. A., Kumar, A. \& Kamath, K. A. (2007). Fecal carriage of extended-spectrum $\beta$-lactamase-producing Escherichia coli and Klebsiella pneumoniae in patients and asymptomatic healthy individuals. Infect Control Hosp Epidemiol 28, 1114-1116.

Kiratisin, P., Apisarnthanarak, A., Laesripa, C. \& Saifon, P. (2008a). Molecular characterization and epidemiology of extended-spectrum$\beta$-lactamase-producing Escherichia coli and Klebsiella pneumoniae isolates causing health care-associated infection in Thailand, where the CTX-M family is endemic. Antimicrob Agents Chemother 52, 2818-2824.

Kiratisin, P., Chattammanat, S., Sa-Nguansai, S., Dansubutra, B., Nangpatharapornthawee, P., Patthamalai, P., Tirachaimongkol, N. \& Nunthanasup, T. (2008b). A 2-year trend of extended-spectrum $\beta$ lactamase-producing Escherichia coli and Klebsiella pneumoniae in Thailand: an alert for infection control. Trans $\mathrm{R}$ Soc Trop Med Hyg 102, 460-464.

Larson, E. (2007). Community factors in the development of antibiotic resistance. Annu Rev Public Health 28, 435-447.

Lautenbach, E., Patel, J. B., Bilker, W. B., Edelstein, P. H. \& Fishman, N. O. (2001). Extended-spectrum $\beta$-lactamase-producing Escherichia coli and Klebsiella pneumoniae: risk factors for infection and impact of resistance on outcomes. Clin Infect Dis 32, 1162-1171.

Lo, W.-U., Ho, P.-L., Chow, K.-H., Lai, E. L., Yeung, F. \& Chiu, S. S. (2010). Fecal carriage of CTXM type extended-spectrum $\beta$-lactamase-producing organisms by children and their household contacts. J Infect 60, 286-292.

Martínez, J. L. (2008). Antibiotics and antibiotic resistance genes in natural environments. Science 321, 365-367.

Monstein, H. J., Ostholm-Balkhed, A., Nilsson, M. V., Nilsson, M., Dornbusch, K. \& Nilsson, L. E. (2007). Multiplex PCR amplification 
assay for the detection of $b l a_{\mathrm{SHV}}, b l a_{\mathrm{TEM}}$ and $b l a_{\mathrm{CTX}-\mathrm{M}}$ genes in Enterobacteriaceae. APMIS 115, 1400-1408.

Moubareck, C., Daoud, Z., Hakimé, N. I., Hamzé, M., Mangeney, N., Matta, H., Mokhbat, J. E., Rohban, R., Sarkis, D. K. \& DoucetPopulaire, F. (2005). Countrywide spread of community- and hospital-acquired extended-spectrum $\beta$-lactamase (CTX-M-15)producing Enterobacteriaceae in Lebanon. J Clin Microbiol 43, 33093313.

Niumsup, P. R., Tansawai, U., Boonkerd, N., Polwichai, P. \& Dejsirilert, S. (2008). Dissemination of extended-spectrum $\beta$-lactamase-producing Klebsiella pneumoniae and Escherichia coli in Thai hospitals. J Infect Chemother 14, 404-408.

Paterson, D. L. \& Bonomo, R. A. (2005). Extended-spectrum $\beta$-lactamases: a clinical update. Clin Microbiol Rev 18, 657-686.

Pitout, J. D. \& Laupland, K. B. (2008). Extended-spectrum $\beta$-lactamase-producing Enterobacteriaceae: an emerging public-health concern. Lancet Infect Dis 8, 159-166.

Pitout, J. D., Hossain, A. \& Hanson, N. D. (2004). Phenotypic and molecular detection of CTX-M- $\beta$-lactamases produced by Escherichia coli and Klebsiella spp. J Clin Microbiol 42, 5715-5721.

Roberts, R. R., Hota, B., Ahmad, I., Scott, R. D., II, Foster, S. D., Abbasi, F., Schabowski, S., Kampe, L. M., Ciavarella, G. G. \& other authors (2009). Hospital and societal costs of antimicrobial-resistant infections in a Chicago teaching hospital: implications for antibiotic stewardship. Clin Infect Dis 49, 1175-1184.

Rodriguez-Baño, J. \& Pascual, A. (2008). Clinical significance of extended-spectrum $\beta$-lactamases. Expert Rev Anti Infect Ther 6, 671-683.
Rodríguez-Baño, J., Navarro, M. D., Romero, L., Muniain, M. A., de Cueto, M., Ríos, M. J., Hernández, J. R. \& Pascual, A. (2006). Bacteremia due to extended-spectrum $\beta$-lactamase-producing Escherichia coli in the CTX-M era: a new clinical challenge. Clin Infect Dis 43, 1407-1414.

Sasaki, T., Hirai, I., Niki, M., Nakamura, T., Komalamisra, C., Maipanich, W., Kusolsuk, T., Sa-Nguankiat, S., Pubampen, S. \& Yamamoto, Y. (2010). High prevalence of CTX-M $\beta$-lactamaseproducing Enterobacteriaceae in stool specimens obtained from healthy individuals in Thailand. J Antimicrob Chemother 65, 666668.

Swartz, M. N. (2002). Human diseases caused by foodborne pathogens of animal origin. Clin Infect Dis 34 (Suppl. 3), S111S122.

Tian, S. F., Chen, B. Y., Chu, Y. Z. \& Wang, S. (2008). Prevalence of rectal carriage of extended-spectrum $\beta$-lactamase-producing Escherichia coli among elderly people in community settings in China. Can J Microbiol 54, 781-785.

Valverde, A., Coque, T. M., Sánchez-Moreno, M. P., Rollán, A., Baquero, F. \& Cantón, R. (2004). Dramatic increase in prevalence of fecal carriage of extended-spectrum $\beta$-lactamase-producing Enterobacteriaceae during nonoutbreak situations in Spain. J Clin Microbiol 42, 4769-4775.

Yuan, L., Liu, J.-H., Hu, G.-Z., Pan, Y.-S., Liu, Z.-M., Mo, J. \& Wei, Y.-J. (2009). Molecular characterization of extended-spectrum $\beta$-lactamase-producing Escherichia coli isolates from chickens in Henan Province, China. J Med Microbiol 58, 1449-1453. 